\title{
Anger Expression: A Study on Gender Differences
}

\author{
$\operatorname{Suman}^{1 *}$
}

\section{ABSTRACT}

Anger or wrath is an intense emotional response. It is a normal emotion that involves a strong uncomfortable and emotional response to a perceived provocation. Often it indicates when one's personal boundaries are violated. Some have a learned tendency to react to anger through retaliation. Anger may be utilized effectively by setting boundaries or escaping from dangerous situations. The present study investigates the gender differences in anger expression. This study investigates that which of the gender express their anger much? For this purpose Spielberger Anger Expression inventory was used. Data was collected from 50 subjects (25 males and 25 females).

Keywords: Anger, Retaliation, Tendency, Gender Differences.

Anger or wrath is an intense emotional response. It is a normal emotion that involves a strong uncomfortable and emotional response to a perceived provocation. Often it indicates when one's personal boundaries are violated. Some have a learned tendency to react to anger through retaliation. Anger may be utilized effectively by setting boundaries or escaping from dangerous situations. Raymond Novaco of UC Irvine, who since 1975 has published a plethora of literature on the subject, stratified anger into three modalities: cognitive (appraisals), somatic-affective (tension and agitations), and behavioral (withdrawal and antagonism). William DeFoore, an anger-management writer, described anger as a pressure cooker: we can only apply pressure against our anger for a certain amount of time until it explodes.

Anger may have physical correlates such as increased heart rate, blood pressure, and levels of adrenaline and noradrenalin. Some view anger as an emotion which triggers part of the fight or flight brain response. Anger becomes the predominant feeling behaviorally, cognitively, and physiologically when a person makes the conscious choice to take action to immediately stop the threatening behavior of another outside force. The English term originally comes from the term anger of Old Norse language. Anger can have many physical and mental consequences.

\footnotetext{
${ }^{1}$ Research Scholar, Rohtak, Jai Bharat High School, Nidana, Jind, Haryana, India *Responding Author

(C) 2016, Suman; licensee IJIP. This is an Open Access Research distributed under the terms of the Creative Commons Attribution License (http://creativecommons.org/licenses/by/2.0), which permits unrestricted use, distribution, and reproduction in any Medium, provided the original work is properly cited.
} 


\section{Anger Expression: A Study on Gender Differences}

The external expression of anger can be found in facial expressions, body language, physiological responses, and at times in public acts of aggression. Animals, for example, make loud sounds, attempt to look physically larger, bare their teeth, and stare. The behaviors associated with anger are designed to warn aggressors to stop their threatening behavior. Rarely does a physical altercation occur without the prior expression of anger by at least one of the participants. While most of those who experience anger explain its arousal as a result of "what has happened to them," psychologists point out that an angry person can very well be mistaken because anger causes a loss in self-monitoring capacity and objective observability.

Modern psychologists view anger as a primary, natural, and mature emotion experienced by virtually all humans at times, and as something that has functional value for survival. Anger can mobilize psychological resources for corrective action. Uncontrolled anger can, however, negatively affect personal or social well-being. While many philosophers and writers have warned against the spontaneous and uncontrolled fits of anger, there has been disagreement over the intrinsic value of anger. The issue of dealing with anger has been written about since the times of the earliest philosophers, but modern psychologists, in contrast to earlier writers, have also pointed out the possible harmful effects of suppressing anger. Displays of anger can be used as a manipulation strategy for social influence.

Three types of anger are recognized by psychologists: The first form of anger, named "hasty and sudden anger" by Joseph Butler, an 18th-century English bishop, is connected to the impulse for self-preservation. It is shared between both human and non-human animals, and it occurs when the animal is tormented or trapped. The second type of anger is named "settled and deliberate" anger and is a reaction to perceived deliberate harm or unfair treatment by others. These two forms of anger are episodic. The third type of anger is called dispositional and is related more to character traits than to instincts or cognitions. Irritability, sullenness and churlishness are examples of the last form of anger.

Anger can potentially mobilize psychological resources and boost determination toward correction of wrong behaviors, promotion of social justice, communication of negative sentiment and redress of grievances. It can also facilitate patience. In contrast, anger can be destructive when it does not find its appropriate outlet in expression. Anger, in its strong form, impairs one's ability to process information and to exert cognitive control over their behavior. An angry person may lose his/her objectivity, empathy, prudence or thoughtfulness and may cause harm to themselves or others. There is a sharp distinction between anger and aggression (verbal or physical, direct or indirect) even though they mutually influence each other. While anger can activate aggression or increase its probability or intensity, it is neither a necessary nor a sufficient condition for aggression. 


\section{Anger Expression: A Study on Gender Differences}

\section{Neuropsychological perspective}

Extension of the Stimuli of the Fighting Reactions. At the beginning of life the human infant struggles indiscriminately against any restraining force, whether it is another human being or a blanket which confines his movements. There is no inherited susceptibility to social stimuli, as distinct from other stimulation, in anger. At a later date the child learns that certain actions, such as striking, scolding, and screaming, are effective toward persons, but not toward things. In adults, although the infantile response is still sometimes seen, the fighting reaction becomes fairly well limited to stimuli whose hurting or restraining influence can be thrown off by physical violence.

\section{Differences between related concepts}

The words annoyance and rage are often imagined to be at opposite ends of an emotional continuum: mild irritation and annoyance at the low end and fury or murderous rage at the high end. Rage problems are conceptualized as "the inability to process emotions or life's experiences" either because the capacity to regulate emotion (Schore, 1994) has never been sufficiently developed or because it has been temporarily lost due to more recent trauma. Rage is understood as "a whole load of different feelings trying to get out at once" (Harvey, 2004) or as raw, undifferentiated emotions, that spill out when another life event that cannot be processed, no matter how trivial, puts more stress on the organism than it can bear.

Anger when viewed as a protective response or instinct to a perceived threat is considered as positive. The negative expression of this state is known as aggression. Acting on this misplaced state is Rage due to possible potential errors in perception and judgement.

One simple dichotomy of anger expression is Passive anger versus Aggressive anger. These two types of anger have some characteristic symptoms:

Passive anger

Passive anger can be expressed in the following ways:

- Dispassion, such as giving someone the cold shoulder or a fake smile, looking unconcerned or "sitting on the fence" while others sort things out, dampening feelings with substance abuse, overreacting, oversleeping, not responding to another's anger, frigidity, indulging in sexual practices that depress spontaneity and make objects of participants, giving inordinate amounts of time to machines, objects or intellectual pursuits, talking of frustrations but showing no feeling.

- Evasiveness, such as turning one's back in a crisis, avoiding conflict, not arguing back, becoming phobic.

- Defeatism, such as setting yourself and others up for failure, choosing unreliable people to depend on, being accident prone, underachieving, sexual impotence, expressing frustration at insignificant things but ignoring serious ones. 


\section{Anger Expression: A Study on Gender Differences}

- Obsessive behavior, such as needing to be inordinately clean and tidy, making a habit of constantly checking things, over-dieting or overeating, demanding that all jobs be done perfectly.

- Psychological manipulation, such as provoking people to aggression and then patronizing them, provoking aggression but staying on the sidelines, emotional blackmail, false tearfulness, feigning illness, sabotaging relationships, using sexual provocation, using a third party to convey negative feelings, withholding money or resources.

- Secretive behavior, such as stockpiling resentments that are expressed behind people's backs, giving the silent treatment or under the breath mutterings, avoiding eye contact, putting people down, gossiping, anonymous complaints, poison pen letters, stealing, and conning.

- Self-blame, such as apologizing too often, being overly critical, inviting criticism.

\section{Aggressive anger}

The symptoms of aggressive anger are:

- Bullying, such as threatening people directly, persecuting, pushing or shoving, using power to oppress, shouting, driving someone off the road, playing on people's weaknesses.

- Destructiveness, such as destroying objects as in vandalism, harming animals, child abuse, destroying a relationship, reckless driving, substance abuse.

- Grandiosity, such as showing off, expressing mistrust, not delegating, being a sore loser, wanting center stage all the time, not listening, talking over people's heads, expecting kiss and make-up sessions to solve problems.

- Hurtfulness, such as violence, including sexual abuse and rape, verbal abuse, biased or vulgar jokes, breaking confidence, using foul language, ignoring people's feelings, willfully discriminating, blaming, punishing people for unwarranted deeds, labeling others.

- Manic behavior, such as speaking too fast, walking too fast, working too much and expecting others to fit in, driving too fast, reckless spending.

- Selfishness, such as ignoring others' needs, not responding to requests for help, queue jumping.

- Threats, such as frightening people by saying how one could harm them, their property or their prospects, finger pointing, fist shaking, wearing clothes or symbols associated with violent behaviour, tailgating, excessively blowing a car horn, slamming doors.

- Unjust blaming, such as accusing other people for one's own mistakes, blaming people for your own feelings, making general accusations.

- Unpredictability, such as explosive rages over minor frustrations, attacking indiscriminately, dispensing unjust punishment, inflicting harm on others for the sake of it, using alcohol and drugs, ${ }^{[23]}$ illogical arguments. 


\section{Anger Expression: A Study on Gender Differences}

- Vengeance, such as being over-punitive. This differs from retributive justice, as vengeance is personal, and possibly unlimited in scale.

One should also consider the context or the bigger picture, e.g an aggressive action of manipulation may in fact work to help an individual, although a contradiction in a PC sense, an important distinction should be made when considering the context of anger when it promotes a positive effect that outweighs the negative. For instance, a display or anger to shock a individual to stop sunbathing intensely, this may save their life, and would be therefore considered socially and morally acceptable in every sense.

\section{Gender Differences in Anger Expression:}

Research indicates that differences exist between adolescent males and females with regard to behavioral decision-making processes and expression of emotions (Brandts \& Garofalo, 2012). Although research depicts females as more emotionally expressive, males have a reputation of being more predisposed to anger. According to Sadeh, Javdani, Finy, and Verona (2011), females experience anger, but may express it differently than males. For example, instead of expressing anger by striking objects, adolescent females may talk to friends or peers (Fischer \& Evers, 2011). Conversely, other studies purport that females express anger similarly to males, but experience difficulty recognizing and admitting the emotion due to social expectations and constraints (Karreman \& Bekker, 2012). Males, on the other hand, tend to display anger more commonly and comfortably (Fischer \& Evers, 2011). One of the many reasons that adolescent males may feel comfortable expressing anger is because it is socially acceptable (Burt et al., 2013).

An extensive number of studies have investigated anger; however, there appears to be a lack of studies exploring anger differences between genders. Karreman and Bekker (2012) conducted a study on gender differences, investigating autonomy-connectedness between genders. Their study indicated differences related to anger and sensitivity between genders. However, the study did not attempt to determine whether males and females were equal in anger at the beginning or end of the study. Similarly, Burt, Patel, and Lewis (2012) reported that incorporating social and relational competencies into anger management groups reduced anger, but there was no discussion of anger differences between genders. Sadeh et al. (2011) indicated that women expressed more self-anger (i.e., anger directed internally toward themselves) than males, but did not investigate whether differences existed between genders before the study.

Although limited, a small number of studies have attempted to examine anger differences between genders. Similar to Sadeh et al. (2011), Fischer \& Evers (2011) found that females expressed subjective anger, or self-anger, more often than males. Buntaine and Costenbader (1997) found that both genders' self-reports (assessments) indicated no significant differences. Upon further examination of their data, however, they concluded that although self-reports 


\section{Anger Expression: A Study on Gender Differences}

specified no differences, males verbally reported higher responses of anger. In contrast, Zimprich and Mascherek (2012) determined that no anger differences existed between males and females. They declared that although genders may express anger and respond to situations differently, they generally experience similar levels of anger. As can be seen from the preceding studies, inconsistences exist in the literature. Contradicting studies indicate that researchers are unclear as to whether differences in anger exist between genders. As such, a research gap has emerged that needs to be filled (Zimprich \& Mascherek, 2012). In order to understand how this research gap developed, it is necessary to examine cultural influences.

\section{Cultural Influences and Misconceptions in Society}

According to Carney, Buttell, and Dutton (2007), a misconception exists in Western society that women are less aggressive than men and do not express excessive anger. This fallacy persisted in Western culture until a report from the U.S. National Family Violence Survey of 1975 (as cited in Carney et al., 2007) found a disturbing trend: Females were just as angry as males and expressed excessive anger the same amount that men did. At the time, feminist theory and the feminist movement were developing and stood in stark contrast to these findings. Carney et al. (2007) stated that as such, the National Family Violence Survey findings were largely unreported, and in extreme situations, people reinterpreted or repudiated the survey's findings. In either case, more misconceptions began to develop in Western culture (Carney et al., 2007), such as the idea that when females experience anger, it is always appropriate to the situation (i.e., anger is permissible). A second mistaken belief is that anger from females is less serious and not as negative. For example, the expression "you look so cute when you're angry” portrays this biased and potentially chauvinistic thought. A third misconception is that females are more credible in reporting their emotions and, as such, females are more reliable when they state that they are not angry.

Western society has acted upon these cultural misconceptions. For example, certain myths in society (and mental health counseling) persist, declaring the following: (a) only males have angry feelings, (b) all male-comprised counseling groups are anger management groups, (c) males have a limited repertoire of emotions to express, (d) males are too angry and competitive to support one another in groups, and (e) males are not interested in meeting with other males (Andronico \& Horne, 2004). Myths about female groups are that they are high functioning, conflicts are resolved faster, and a fair amount of reflection and processing exists (Gladding, 2012). According to researchers, these misconceptions can bias the truth regarding people's beliefs. For example, Winstok (2011) stated that rates of excessive anger and intimate partner physical abuse among females equal or surpass those of males.

Clearly, cultural misconceptions of gender differences in excessive anger can lead mental health counselors to do a disservice to males and females alike. For example, culture can influence mental health and group counseling by causing a type to develop. This type is defined as best 


\section{Anger Expression: A Study on Gender Differences}

suited to be in anger management groups. As a result, mental health counselors may unconsciously choose more males than females to be members of anger management groups. Thus, a population that desperately needs services can go without an intervention (Carney et al., 2007). Mental health counselors need to reevaluate their thinking in order to avoid overlooking a population needing services due to implicit social misconceptions.

Bandura (2008a) believed that excessive anger was not sudden, but gradually manifested over time. His studies with youth corroborated this idea, as he observed modeling and negative behavioral patterns leads to excessive anger (Bandura, Ross, \& Ross, 1963). Supporting Bandura's work, Burt and Butler (2011) asserted that excessive anger begins in childhood and adolescence. They reinforced the notion that mental health counselors must be aware that both genders have common needs and issues. For females, not receiving services or having services denied, and being told that the emotion they feel is inappropriate, could cause personal damage (Gottfredson, 2002). For instance, society and mental health counselors often depict males as more in need of anger management (Burt \& Butler, 2011). Conversely, mental health counselors sometimes neglect and ignore what females need (West-Olatunji et al., 2010). Stated succinctly, a gap exists between what clients need and the options mental health counseling interventions offer to both genders. It is the author's contention that this gap is an unfair practice, as both genders have similar needs. Research has shown that males and females experience anger equally; as a result, both need anger management groups.

To determine whether both genders expressed anger similarly, the author implemented this study with adolescents to explore the topic As Bandura (2008b) pointed out, anger begins early in life and timely prevention is critical. Provision of early services for children and adolescents can help to prevent issues later in life.

\section{Problem:}

1. To determine whether both genders express anger similarly.

\section{Hypothesis:}

2. State and trait anger would vary significantly between males and females.

3. Females would be high on suppressing (anger in) their anger than males.

4. Anger expression and all its components would vary significantly between males and females.

\section{METHODS}

\section{Sample:}

50 subjects (25 males \& 25 males), age range: 20-30 years were selected on the basis of availability. All subjects were at least graduates. 


\section{Anger Expression: A Study on Gender Differences}

\section{Tools:}

Self rating questionnaire was used to measure the anger in the subjects. This test was made by Spielberger in 1961. There are 44 questions in this scale. Ten questions are related to trait anger where as ten questions are related to state anger. Twenty four are related to anger expression. Each statement is to be rated by the respondent on a four point scale.(not at all to very much). For scoring, one mark is given to not at all, two marks for somewhat, three marks for moderately and four marks for very much answers. Trait and state scores are computed by adding the scores on each respective sub scale. The scores for anger expression is further subdivided into three parts i.e. anger in, anger out and anger control. Score for anger expression is computed by this formula: Anger in + Anger out- Anger control+16

\section{Procedure:}

The test was administered at the teaching institutes. Respondents were students, teachers and non teaching staff members. Administration of the test was done in a group setting(maximum number of respondents in one group was five.) Following instruction were given to the subjects. "you will be given a booklet and a rating sheet. Initially ended your name, sex, age, date, education and occupation in the space provided on this booklet and on the top of the rating sheet. This booklet is divided into three parts. Each part contains a number of statements that people use to describe their feeling and behaviour. Please note that each part has different directions. Carefully read the directions for each part before recording your responses on the rating sheet. There is no right or wrong answers. In responding to each statement, give the answer that suits you.” After giving the instructions, booklet and rating sheet were giver to the subjects. When all the questions had been answered, the test booklet and respondent sheet was taken from the respondents. Scoring was done with the help of the scoring key. Same procedure was repeated for the remaining respondents.

\section{RESULTS AND DISCUSSION}

The main aim of the study was to study the gender differences in anger. For this purpose, data of 50 subjects were collected.

Table 1 shows the mean scores of males and females on anger expression

\begin{tabular}{|l|l|l|l|}
\hline Variable & Sub part & Females & Males \\
\hline State anger & & 15.08 & 13.2 \\
\hline Trait anger & & 17.48 & 17.72 \\
\hline Anger expression & & & \\
\hline & Anger in & 16.36 & 15.8 \\
\hline & Anger out & 13.24 & 20 \\
\hline & Anger control & 20.64 & 24.2 \\
\hline
\end{tabular}




\section{Anger Expression: A Study on Gender Differences}

On the basis of the above data it was found that the mean scores of the females is high on state anger, where mean score of males is high on trait anger. These shows that female perceive wider range of situations as anger provoking (e.g. annoying frustrating) than males.

On anger expression, the mean score of females is 20.64 and the mean scores of males are 24.2. This difference show that males express their anger on the other hand females suppress their anger. Further consideration of the sub parts shows that on anger in , the mean scores of females is 16.36 and the mean scores of males is 15.8. On anger out, the mean scores of females is 13.24 and the mean score of female is 15.8. Thus both results prove that male express their anger. These results indicate the mean score of males is higher on anger expression than females. In order to determine whether the difference in the mean scores of the males and females were significant, t test was applied.

Table: 2 showing the $t$ - test scores of males and females

\begin{tabular}{|l|l|}
\hline Variables & t value \\
\hline State anger & 1.634 \\
\hline Trait anger & .0102 \\
\hline Anger in & 4.098 \\
\hline Anger out & 2.84 \\
\hline Anger control & 3.36 \\
\hline
\end{tabular}

On state anger the mean score of female subjects is 15.08 and mean score of male subject is 13.2. The value of $t$ is 1.0634. This shows that there is no significant difference in two means. On trait anger the mean score of female subjects is 17.48 and the mean scores of male subjects is 17.72. The value of $t$ is 0.0102 which is again not significant. These results verify the first hypothesis which predicted that state and trait anger would not vary significantly in males and females is verified. These results receive support from a number of earlier studies. Winstok (2011) also found the same result in their study. In order to consider the gender variation in Anger expression the mean scores of males and females were compared. The Anger in mean score of females is 16.36 and mean scores of males is 12.52 . The value of $t$ is 4.098 . This shows that there is a significant difference in these two means. This indicates that females direct their anger in more than males do.

On angers out, mean scores of males is 20 and mean score of female is 24 . The value of $t$ is 3.36 . Since the mean score of males is higher. It shows that males direct their anger outward. On anger control, the mean score of males of males is 20 and mean score of female is 24 . The value of $t$ is 3.36. Considered together the present studies shows that trait and state anger does not differ across gender, but expression of anger is gender specific. These results implicate a role of social learning in expression of anger. Since similar results have been reported in different cultural settings (western countries, various ethnic groups.) thus it can be concluded that females express anger less in spite of males.

(C) The International Journal of Indian Psychology, ISSN 2348-5396 (e)| ISSN: 2349-3429 (p) | 62 


\section{Anger Expression: A Study on Gender Differences}

\section{Acknowledgments}

The author appreciates all those who participated in the study and helped to facilitate the research process.

\section{Conflict of Interests}

The author declared no conflict of interests.

\section{REFERENCES}

Brandts, J., \& Garofalo, O. (2012). Gender pairings and accountability effects. Journal of Economic Behavior \& Organization, 83, 31-41. doi:10.1016/j.jebo.2011.06.023

Buntaine, R. L., \& Costenbader, V. K. (1997). Self-reported differences in the experience and expression of anger between girls and boys. Sex Roles, 36, 625-637. doi:10.1023/A:1025670008765

Burt, I. (2010). Addressing anger management in a middle school setting: Initiating a leadership driven anger management group (Doctoral dissertation, University of Central Florida). Retrieved from http://etd.fcla.edu/CF/CFE0003375/Burt_Isaac_201008_PhD.pdf

Burt, I., \& Butler, S. K. (2011). Capoeira as a clinical intervention: Addressing adolescent aggression with Brazilian martial arts. Journal of Multicultural Counseling and Development, 39, 48-57. doi:10.1002/j.2161-1912.2011.tb00139.x

Fischer, A. H., \& Evers, C. (2011). The social costs and benefits of anger as a function of gender and relationship context. Sex Roles, 65, 23-34. doi:10.1007/s11199-011-9956-X

Geddes, D. \& Callister, R. 2007 Crossing The Line(s): A Dual Threshold Model of Anger in Organizations, Academy of Management Review. 32 (3): 721-746.

Georges Bernanos, The Diary of a Country Priest (London: Fontana Books, 1956), 126-150 passim.

Graham, Michael C. (2014). Facts of Life: ten issues of contentment. Outskirts Press. ISBN 9781-4787-2259-5.

Graham, Michael C. (2014). Facts of Life: ten issues of contentment. Outskirts Press. p. 73. ISBN 978-1-4787-2259-5.

Henri J. M. Nouwen, "Forward" in May I Hate God? Pierre Wolff, 2 (Paulist Press, 1979).

Karreman, A., \& Bekker, M. H. J. (2012). Feeling angry and acting angry: Different effects of autonomy-connectedness in boys and girls. Journal of Adolescence, 35, 407-415. doi:10.1016/j.adolescence.2011.07.016

Raymond W. Novaco, Anger, Encyclopedia of Psychology, Oxford University Press, 2000

Sadeh, N., Javdani, S., Finy, M. S., \& Verona, E. (2011). Gender differences in emotional risk for self- and other-directed violence among externalizing adults. Journal of Consulting and Clinical Psychology, 79, 106-117. doi:10.1037/a0022197

St. Thomas Aquinas Blackfriars; McGraw-Hill, N. Y. K. 1963, Question 158

Videbeck, Sheila L. (2006). Psychiatric Mental Health Nursing (3rd ed.). Lippincott Williams \& Wilkins. 


\section{Anger Expression: A Study on Gender Differences}

Zimprich, D., \& Mascherek, A. (2012). Anger expression in Swiss adolescents: Establishing measurement invariance across gender in the AX scales. Journal of Adolescence, 35, 1013-1022. doi:10.1016/j.adolescence.2012.02.008

How to cite this article: Suman (2016), Anger Expression: A Study on Gender Differences,

International Journal of Indian Psychology, Volume 3, Issue 4, No. 65, ISSN 2348-5396 (e), ISSN: 2349-3429 (p), DIP: 18.01.140/20160304, ISBN: 978-1-365-34680-4 